\title{
Tangence
}

\section{L'interférence acadienne dans la lecture des Portes tournantes de Jacques Savoie}

\section{Pierre L'Hérault}

Numéro 58, octobre 1998

Le postmoderne acadien

URI : https://id.erudit.org/iderudit/025981ar

DOI : https://doi.org/10.7202/025981ar

Aller au sommaire du numéro

Éditeur(s)

Tangence

ISSN

0226-9554 (imprimé)

1710-0305 (numérique)

Découvrir la revue

Citer cet article

L'Hérault, P. (1998). L'interférence acadienne dans la lecture des Portes

tournantes de Jacques Savoie. Tangence, (58), 5-7.

https://doi.org/10.7202/025981ar d'utilisation que vous pouvez consulter en ligne.

https://apropos.erudit.org/fr/usagers/politique-dutilisation/ 


\section{L'interférence acadienne dans la lecture des Portes tournantes de Jacques Savoie}

\section{Pierre L'Hérault}

Le roman de Jacques Savoie, Les portes tournantes ${ }^{1}$ met en place une double stratégie de lecture présupposant une double compétence lectoriale: québécoise et acadienne. On peut penser que cette double compétence est attendue du "Lecteur Modèle" prévu. Mais la particularité du roman de Savoie vient justement de ce qu'il peut se lire sans que ces deux compétences soient réunies en un même lecteur. On pourrait en conséquence entrevoir quatre positions lectoriales. D'abord celle du lecteur québécois "moyen", c'est-à-dire sans connaissance spécifique de l'Acadie; il pourra faire une lecture des Portes toumantes sans voir la référence acadienne, cette dernière se trouvant non seulement désactivée mais à servir de renforcement à la référence québécoise. Ensuite bien sûr celle du lecteur acadien "moyen"; il y repérera au contraire sans difficulté une série de signes et d'indices qui, réactivant le drame acadien, ne pourront que le conduire à accorder spontanément préséance à la référence acadienne, la référence québécoise devenant secondaire, s'abolissant même dans la référence acadienne dans laquelle elle s'inclut comme lieu de déportation. À cet égard, on pourrait dire que la particularité du roman de Savoie aura été d'offrir en un seul texte deux romans distincts à deux lectorats étanches. Mais l'intérêt des Portes toumantes ne s'arrête pas là. Savoie ne viserait-il pas en définitive, en brisant cette étanchéité, un lecteur hybride? Il faut alors penser à deux autres lecteurs: un lecteur québécois-acadien et un lecteur acadien-québécois ${ }^{2}$. Dans ce croisement de compé-

1 Jacques Savoie, Les pontes toumantes, roman, Montréal, Boréal, 1984. Je renverrai à ce texte par une simple mention de la page entre parenthèses.

2 C'est à dessein que je forme ce chiasme sur le modèle de celui de Mikhail Bakhtine - mien-étranger/étranger-mien* (Estbétique de la création verbale, Paris, Gallimard, 1979, p. 365), car le rapport de l'* étranger* au * mien * est ici tout à fait pertinent. - Au sujet du quatrième lecteur, je me suis demandé si son existence n'était pas purement théorique. Alors qu'on peut très bien imaginer un lecteur québécois sans compétence acadienne, il est difficile, notamment à cause de l'importante diaspora acadienne montréalaise, d'imaginer un lecteur acadien qui n'aurait pas de compétence québécoise. 
tences le roman devient le lieu d'une série d'interférences des discours imaginaires et culturels acadien et québécois qui contribuent à "élargir l'univers de discours " 3 du lecteur.

Si je parle d'"interférence acadienne " ${ }^{4}$ à propos de la lecture d'un texte de Jacques Savoie, originaire d'Acadie, c'est en premier lieu que je suppose ce texte "québécois". Qu'il soit publié par une maison québécoise, Boréal, n'implique-t-il pas qu'il vise d'abord un lectorat québécois? Mais c'est aussi que je ne peux lui dénier une certaine acadianité, ne serait-ce qu'à cause de cette information biographique concernant l'auteur contenue en quatrième de couverture: "Né en Acadie en 1951". Une telle mention suggère la possibilité d'une lecture acadienne concurrente à la lecture québécoise. Mais si je parle d'«interférence acadienne", c'est en définitive parce que ma compétence est celle d'un lecteur "québécoisacadien". C'est-à-dire d'un lecteur québécois possédant une (certaine) connaissance intellectuelle et affective de l'Acadie $^{5}$. Si cette position est tout à fait personnelle et pragmatique, la seule possible en fait, il me faut reconnaitre qu'elle est privilégiée. Car si le romancier n'exige pas de son lecteur acadien ou québécois une double compétence, offrant plutôt à chacun une histoire lisible à partir de sa compétence particulière, il n'en demeure pas moins que le "sort interprétatif ${ }^{6}$ de son texte est mieux servi par la double compétence lectoriale. En ce qui me touche en tout cas mais puis-je parler d'un autre lieu que celui-ci? - ce que j'appelle l' «interférence acadienne" comble ma lecture en faisant intervenir dans mon espace imaginaire les "mouvements de l'autre " 7 prévus

3 Umberto Eco, Lector in fabula. Le rôle du lecteur, Paris, Grasset, coll. "Livre de poche", 1979, p.74.

4 Un premier état de cette réflexion a été proposé sous forme de communication au colloque conjoint (UQAM, Université de Western, de Toronto et Concordia) - Les interférences •, tenu à l'Université Concordia les 11 et 12 novembre 1994 .

5 J'ai vécu quatre ans (1972-1976) en Acadie, comme professeur à l'Université de Moncton, où j'ai d'ailleurs rencontré mon épouse. À l'appui de ma compétence intellectuelle je citerai, parmi d'autres, les travaux suivants: l'importante section (*III. L'extrême frontière acadienne *) de mon étude *Pour une cartographie de l'hétérogène: dérives identitaires des années 1980", S. Simon, P. L'Hérault, R. Schwartzwald, Alexis Nouss (dir.), Fictions de l'identitaire au Québec, Montréal, XYZ, 1991; et -Préface" de Cri de terre de Raymond (Guy) LeBlanc, édition revue et corrigée, Moncton, Éditions d'Acadie, 1992

6 Umberto Eco, op. cit., p. 65. 
68

par la stratégie textuelle. Or, quand il s'agit de l'Acadie, les mouvements de l'autre viennent nécessairement remettre en cause les cartographies de l'appartenance.

\section{Un récit québécois}

Je reconnaîtrai dans un premier temps que le roman de Savoie se lit sans référence à son acadianité. L'histoire se situe dans la ville de Québec, évoquée avec suffisamment de précision et cle réalisme pour constituer un espace reconnaissable par le lecteur et comme le seul espace réel des personnages. Parmi les lieux caractéristiques et fonctionnels, notons le Grand Théâtre où se dénoue le roman, les Plaines d'Abraham, assiduement fréquentées par le personnage principal, les noms des rues et des côtes empruntées par les personnages... Même la référence acadienne contenue dans le patronyme "Beaumont" peut être détournée au profit de la référence québécoise, puisqu'il est aussi un vieux toponyme de la région de Québec. Si l'espace est québéquois, le temps l'est aussi. Le temps du récit est celui d'un homme de quarante ans, Blaudelle, peintre de son métier, qui traverse une crise d'identité ayant des effets sur ses proches: sa femme Lauda l'a laissé depuis quelque temps; son fils de dix ans, Antoine, vit avec lui, et cherche sa place dans cet espace parental conflictuel. Mais le passé intervient dans le présent du récit. Un jour Blaudelle reçoit une liasse de lettres, celles de sa mère Céleste Beaumont dont il a été séparé peu après sa naissance. Ces lettres lui permettront de retracer l'histoire de sa mère, pianiste de cinéma muet, à partir de Val d'Amour et de Campbellton, au Nouveau Brunswick, jusqu'à New York, où elle meurt en 1945, en passant par Montréal, et Petawawa. S'il y a remontée vers l'origine, il ne faut pas donner à cette remontée une dimension collective (recherche de l'appartenance acadienne), mais lui garder sa dimension individuelle. Le passage suivant montre que l'histoire de "la petite pianiste " n'a valeur d' épopée" que pour son fils qui peut y retrouver ce qui lui manquait de son histoire:

Ma mère est morte à New York en 1945, sans savoir que la guerre était finie. Elle est morte en confondant la guerre et l'hiver. Trente ans plus tard, sans le savoir, j'ai achevé le travail qu'elle avait commencé dans le clan Blaudelle. Je suis devenu

7 Ibid. Forçons un peu du mot • autre " pour le pousser vers * étranger *. 
l'artiste qu'on ne voulait pas qu'elle soit. On m'a renié, comme on l'avait reniée, elle, et j'ai dû moi aussi déserter.

L'épopée de la petite pianiste de cinéma de Campbellton m'a rejoint. Quand je relis ses lettres, j'ai l'impression d'arriver au bout d'une très longue course, au bout d'un marathon que je courais depuis toujours sans le savoir. (p. 135)

La rencontre du présent et du passé n'excède pas le cadre des retrouvailles, par delà la mort, du fils et de la mère et ne débouche pas sur un retour symbolique à la patrie, à la matrie acadienne. Le lieu d'appartenance, Québec, n'est jamais remis en cause par Blaudelle et n'est pour rien dans sa crise d'identité ${ }^{8}$. Notons à ce propos que l'action du roman de Savoie se déroule non dans l'espace mobile, hybride, migratoire de Montréal - qui est aussi un important espace diasporique acadien -, mais dans l'espace de Québec dont la géographie est un constant renvoi à la fixité et à l'homogénéité originelles. Comparé à cet espace, l'espace évoqué par les souvenirs des "promenades dans le parc de Campbellton" est évanescent, irréel, comparé à celui de Québec: "Avec Antoine, sur les Plaines d'Abraham, je me tiens debout et bien droit devant mon chevalet [...]" (p. 135) L'espace d'origine de la mère n'entre donc pas en contradiction avec l'espace habité par Blaudelle: il ne définit pas un "pays" d'origine, mais l'origine de Blaudelle. Céleste n'est pas un symbole, mais une mère. Et Blaudelle ne cherche pas en elle autre chose: " Ma mère [...]"

Il manque à Blaudelle le début de son histoire, non une Histoire à laquelle il aurait été arraché. Blaudelle n'est ni un exilé, ni un déporté. Quand à la fin, les fils de l'histoire auront été renoués, grâce à l'intervention de Devil, jamais la question du retour, de la nostalgie d'un paradis perdu, de l'origine acadienne maternelle, ne sera évoquée. La rencontre se fera à l'intérieur du Grand théâtre de Québec que Devil quittera, après avoir permis la rencontre posthume avec la mère, laissant à Blaudelle tout son espace. Les espaces de la mère et du fils sont bien délimités.

Je n'invente pas cette lecture. La possibilité d'une lecture des Portes toumantes sans référence à l'Acadie est confirmée de façon

8 L'importante place accordée à l'enfant, Antoine, dans la narration confirme ce point de vue. C'est d'une façon très directe et personnelle qu'il saisit et décrit les choses. 
70

unanime par la réception critique québécoise. Gilles Dorion y "retrace la vie d'une famille tiraillée entre l'amour et la musique", famille composée d'un garçon de dix ans, Antoine, "dont le destin semble d'apprendre la musique et qui vit aux côtés d'un père peintre [...] séparé depuis peu de sa femme Lauda" et depuis toujours de sa mère Céleste qu'il retrouve par les "lettres contenues dans un livre noir" reçu de manière inattendue 9 . Quand on mentionne l'Acadie, c'est pour dire qu'elle ne s'y trouve pas. Ainsi François Hébert écrit que Jacques Savoie nous laisse entendre qu'on peut "quitter [l'Acadie], l'oublier et vivre intensément ailleurs" 10. Réginald Martel va plus loin: il ne reconnait plus dans le personnage de Savoie le "héros de la littératue acadienne". "Jacques Savoie a fait le pas"11, conclut-il. Autre indice de cette lecture sans référence à l'appartenance acadienne: c'est à Jacques Poulin, l'un des écrivains québécois de sa génération qui s'est tenu le plus en marge du "roman national", que le comparent Dorion et Martel, et cela avant qu'Une bistoire de cour ${ }^{12}$ vienne rendre ce rapprochement plus évident, ainsi qu'en témoignera le compte rendu de Gilles Marcotte ${ }^{13}$. Ainsi donc, sous le rapport de la dimension collective de l'identité, on pourrait parler d'une lecture "neutre", aussi bien du point de vue québécois que du point de vue acadien, en ce sens que les signes de l'identité québécoise et acadienne sont neutralisés au profit de l'identité individuelle.

\section{L'interférence acadienne}

Cette lecture neutre de la critique québécoise est-elle possible pour un lecteur acadien ou un lecteur possédant une certaine compétence acadienne? Il me semble que non, tellement il est vrai qu'un certain nombre de signes renvoient spontanément au vieux récit de la cléportation. Il y a dans le roman de Savoie, habilement disposés et dosés une série de signes qui, moyennant une

9 Gilles Dorion, - Les portes tournantes [de] Jacques Savoie", Québec français, mai 1984, p. $7-8$.

10 François Hébert, "Un livre qui sonne juste, une découverte , Le devoir, 7 avril 1984 , p. 21.

11 Réginald Martel, "Les portes toumantes. La séduction selon Jacques Savoie", La presse, 24 mars 1984, p. D-3.

12 Jacques Savoie, Une bistoire de coutr, roman, Montréal, Boréal, 1988.

13 Gilles Marcotte, "Un demi-roman de Jacques Savoie, L'actualité, janvier 1989, p. 116. 
connaissance minimale de l'Acadie, conduisent à voir dans l'histoire de Blaudelle et de sa mère une "actualisation" du récit acadien, s'écartant cependant du modèle canonique auquel pourrait ramener une lecture simplificatrice et allégorique, tentante il est vrai tellement le "destin" de Céleste peut recouper celui d'Évangéline: Céleste Beaumont quitte Val d'Amour pour Campbellton où elle est violée par son patron anglais Litwin; elle vit une relation maritale difficile avec Pierre Blaudelle, fils d'une famille riche et puissante (figure de la domination anglaise?), avec qui elle aura un enfant mais dont elle sera séparée par la bellefamille; elle erre finalement sur les routes d'Amérique: Petawawa, Montréal et New York où elle meurt auprès d'un jazzman noir.)

Le nom de la mère de Blaudelle, Céleste Beaumont est l'un de ces signes, et sans doute le plus important qui peut même servir de fil d'Ariane. Si "Beaumont" est un toponyme familier aux Québécois, surtout ceux de la région cle Québec, il évoque pour les Acadiens un lieu à haute teneur symbolique, presque mythique, voire mystique. Formant un petit promontoire, il est situé sur un rétrécissement de la Baie de Shepody par où s'enfoncent dans les terres jusqu'à Moncton les fameuses marées de la Baie de Fundy provoquant sur la rivière Petitcodiac le spectaculaire refoule. Sur ce promontoire, il y a une petite chapelle qui marque le bout du monde. On en fait le tour et on repart en sens inverse, à moins d'emprunter un chemin forestier peu invitant. Bout et origine du monde. Car, pour y accéder la route traverse Pré-d'en Haut, localité ainsi nommée parce qu'il s'agit bien d'un de ces prés construit par les Acadiens à même les marécages, grâce au système d'aboiteaux qui bloquaient l'entrée d'eau, aux marées montantes, et qui permettaient l'écoulement des surplus, aux marées basses. Nous sommes donc en quelque sorte ramenés aux sources historiques et imaginaires de l'Acadie qui, dès sa fondation, a pratiqué cet art de récupérer des terres.

Et les écrivains du renouveau des années soixante-dix n'ont pas manqué de valoriser la symbolique de ce lieu. Raymond-Guy LeBlanc, par exemple, dans un poème de Cri de terre, adressé aussi bien à l'Acadie qu'à l'amante, écrit: "Tu es mon Pré-d'enHaut ma colline vivante. "14 Jacques Savoie lui-même a

14 Raymond (Guy) LeBlanc, Cri de terre, poèmes, [1972], édition revue et corrigée; préface de Pierre L'Hérault; suivi d'une analyse critique de Murielle Béliveau, Moncton, Éditions d'Acadie, 1992, p. 49. 
commencé sa carrière d'écrivain en s'y référant. Son Anti-livre ${ }^{15}$, publié en collaboration avec Herménégilde Chiasson contient une référence encore plus concrète au Pré-d'en-Haut. L'anti-livre se présente sous la forme d'un coffret contenant des feuillets de poèmes polycopiés et quelques herbes de Pré-d'en-Haut. Comme à l'époque on fumait allègrement une certaine herbe, celle de Pré-d'en-Haut rappelait que l' Acadie chaude " 16 des années soixante-dix ne se ressourçait pas qu'à ses origines mythiques, mais aussi à la contre-culture. À travers ce patronyme de Beaumont, Savoie se rattache donc aux origines même de son écriture qui participe à la définition d'une nouvelle acadianité.

Beaumont établit d'une autre façon le rapport à l'origine. Sa chapelle constituait en effet une desserte pour les Amérindiens. À côté se trouve toujours l'ancien cimetière amérindien. Comment ne pas retrouver alors dans le roman de Savoie, par ce détour à Beaumont, cette "conscience amérindienne" de l'Acadie dont Chiasson, à l'instar de Régis Brun ${ }^{17}$, mais sous un autre mode, fait état dans sa préface à L'extrême frontière de Gérald Leblanc?

La célèbre phrase de Ronald Després me revient soudain à l'esprit : ‘ À quoi bon? Où irions-nous? Notre empire est incertain comme l'eau. "Incertain, mais aussi craintif d'affirmer comme sur l'eau notre royaume où il n'y a plus de frontières, et ainsi de balayer du revers de la main ce vieux rêve d'Europe, en propriétaire et arpenteur de l'univers. Notre conscience amérindienne n'a jamais été à l'aise dans cette chimère et nous savons que tôt ou tard il faudra en arriver à nous remettre en accord avec une notion plus étendue et plus équilibrée du territoire. Dans les textes de Gérald Leblanc, je reconnais cette conscience aiguë de l'errance amérindienne, une errance qui est peut-être notre seule dimension possible, mais l'Acadie nous appelle comme une mère qui pleure, et cet appel est déchirant à plus d'un point de vue. Pour nous, après avoir dénoncé le rôle clu père, il restera toujours le rôle de la mère, la conscience de la langue maltraitée, de la terre violée et volée, de la vie invivable. ${ }^{18}$

15 Jacques Savoie, L'anti-litre, poésie, Moncton, Les Éditions de l'Étoile magannée, 1972. Collaboration de Herménégilde Chiasson et Gilles Savoie. (Texte polycopié)

16 L'expression est de Gérald Leblanc et Claude Beausoleil, dans La poésie acadienne 1948-1988, Trois-Rivières, Écrit des Forges/Le Castor Astral, p. 7.

17 Régis Brun, La Mariecomo, Montréal, Éditions du jour, 1974.

18 Herménégilde Chiasson, •Pour saluer Gérald Leblanc *, dans Gérald Leblanc, L'extrême frontière. Poèmes 1972-1988, Moncton, Éditions d'Acadie, 1988, p. 12, 
Chiasson fait un autre rapprochement qui intéresse la lecture des Portes tournantes. Il s'agit du rapprochement entre Acadiens et Noirs américains: "On peut établir des liens entre la situation des Acadiens et celle des Noirs américains parce qu'ils sont errants; ils n'ont pas de territoire défini. Il y a aussi une affinité au niveau du destin. Les complaintes noires, les blues, sont nés après la déportation des noirs." 19 Dans le roman de Savoie, ces liens prennent la figure très concrète de John Devil, encore appelé Papa John, jazzman noir de New York, qui a tiré Céleste de son désespoir montréalais en l'amenant dans la métropole américaine finir sa vie auprès de lui. Ce n'est pas fortuitement que le cahier où sont réunies les lettres de la mère s'appelle le Livre noir. Il est important de noter ici que Devil n'est pas simplement quelqu'un dont on parle. Il est un personnage qui joue un rôle déterminant dans le roman puisqu'il est le seul lien physique entre Blaudelle et sa mère disparue. C'est au cours du concert qu'il donne au Grand Théâtre que tous les fils de l'histoire se nouent, que l'identification et l'identité sont établies de part et d'autre: "- Are you the Devil? [...] - Are you the boy? I mean, are you Céleste's son?" (p. 156-157) C'est dans l'espace de Devil, l'espace du jazz, l'espace du noir - à la faveur d'une panne d'électricité qui plonge le Grand théâtre dans le noir - que la lumière se fait pour Blaudelle. Dans ce jeu des oppositions du noir et du blanc, de l'obscurité et de la clarté, de Devil et de Céleste, où s'inversent volontiers les symboliques, il y a un rappel du mythe de la déportation qu'on ne saurait justifier uniquement par le fait que la figure de Devil évoquerait la Louisiane, pays des Noirs, du jazz et des Acadiens qui, comme les Noirs, furent privés de certains droits ${ }^{20}$. Mais, il faut voir encore, et surtout dans le

On peut en effet lier cette réflexion de Chiasson à sa lecture de Gérald Leblanc dont le titre du recueil qu'il préface est en référence explicite à la géographie amérindienne. Dans le poème intitulé "géographie de la nuit rouge ", on peut lire: «sur la réserve dont Moncton est l'extrême frontière / [...]/ et la / nuit toujours la nuit que j'aime rouge. sur ce continent, / à même la terre amérindienne, souterrain sensible de / notre mémoire ignée.* (p. 65)

19 Pierre-André Arcand, "Imposer la sensation *, Si que II (numéro spécial de la Revue de l'Université de Moncton, vol. VII, n $\mathrm{n}^{\circ}$, mai 1975, p.132-133.

20 L'historien Léon Thériault écrit : •[...] l'Acadie, on la voulait morte. En 1758 , une loi de la Nouvelle-Écosse interdisait à tout "papiste" [...] le droit à la propriété. Il faudra attendre jusqu'en 1783 pour que cette loi, également appliquée au Nouveau-Brunswick actuel, soit amendée dans un sens plus favorable aux catholiques et ce, surtout à la demande des Irlandais. Quant aux droits scolaires, une loi de la Nouvelle-Écosse promulguée en 1766 
74

rapprochement des noms Céleste et Devil une ironie démystificatrice du mythe d'Évangéline qui retrouve un Gabriel plutôt inattendu dans une errance qui n'a rien de la patrie définitive promise par le nom de Céleste. C'est le diable (Devil) qui jouera la musique (de) Céleste:

- For my next number, I'd like to play this piece of music written by a very fine musician from your country. Although she bas passed away now. You dont't kill a piano player will remain one of the most brilliant jazz composition of the Fourties. For you all and specially for Miss Céleste who wrote it... (p. 152)

Mais, a-t-on remarqué que l'instrument dont joue Papa John, c'est le violon, l'instrument traditionnel acadien, qui est aussi, dans le folklore, celui du diable? Si l'on peut reconnaître dans l'histoire de Céleste le scénario acadien de la déportation, il importe de noter l'interprétation qui lui est donnée par Céleste et Blaudelle. Céleste parle d'elle non comme d'une déportée, mais comme une "déserteuse" (p. 41), terme que Blaudelle reprendra à son compte: "[...] et j'ai dû moi aussi déserter. (p. 135) La conscience de Blaudelle rejoindrait alors celle qui s'exprime à travers l'expression de Serge Patrice Thibodeau: "assumer sa déportation / ouvre grand les portes" 21 ou la suggestion de Chiasson que "nous pourrions vivre à la "mesure de notre imaginaire" [de déportés]" 22 . Le lecteur acadien pourra-t-il éviter de reconnaitre en Blaudelle un Acadien de la diaspora qui le renvoie à une conscience fondée sur la double expérience et la double mémoire de l'enracinement et du déracinement, du rattachement à un territoire et d'un éloignement de ce territoire? Cela se traduirait dans Les portes tournantes par un rappel du scénario de la déportation, mais vidé de son sens mythique.

La déportation réapparaît donc sous une forme on ne peut plus moderne qui est une conscience critique et inquiète interrogeant l'appartenance fondée sur une exclusivité territoriale, sur

interdisait à tout "papiste" de fonder une école sous peine d'amende et de prison. C'est en 1786 seulement qu'une loi plus tolérante sera votée. Du point de vue des droits politiques, les catholiques furent exclus du droit de vote jusqu'en 1789 en Nouvelle-Écosse et jusqu'en 1810 dans les autres provinces. (La question du pouvoir en Acadie, Moncton, Éditions d'Acadie, 1982, p. 24-25)

21 Serge Patrice Thibodeau, La septième cbute. Poésie 1982-1989, Moncton, Éditions d'Acalie, 1990, p.107.

22 Herménégilde Chiasson, op. cit., p. 11. 
une chimère européenne, comme le disait Chiasson. Les personnages de Savoie se déplacent dans l'espace américain comme dans un espace familier. Cela est confirmé d'ailleurs par son deuxième roman Une bistoire de coeur. La déportation, dégagée de son sens mythique et mystique apparaît comme une aptitude à traverser les frontières, à les rendre plus fragiles, plus "extrêmes", selon l'expression de Gérald Leblanc dont la poésie d'ailleurs traverse l'espace "multipiste" 23 des mots américains, sans se soucier des frontières.

\section{Masquer l'acadianité}

C'est bien cette conscience de la modernité et de l'universalité que Savoie tient à mettre de l'avant, plutôt que son acadianité au point de nier la présence de cette dernière dans ses écrits, ainsi qu'on peut s'en rendre compte dans un publireportage de La presse:

Jacques Savoie est acadien d'origine, mais il ne cherche pas à l'être dans ses écrits. Il ne le renie pas. Cela fait simplement partie de son histoire, comme ses études à Aix-en-Provence ou son expérience musicale au sein du groupe Beausoleil-Broussard. Mais il a résolument opté pour la modernité et l'universalité.

C'est pourquoi il est si heureux que les premières du film Les Portes toumanles, aient lieu en même temps à Campbellton, petite ville acadienne où fictivement l'action clu film se passe, la ville à ressourcer, et à Paris, la grande capitale française, la ville à conquérir. ${ }^{24}$

Dans Le devoir du $1^{\text {er }}$ octobre 1988 , tout en poussant très loin l'identification entre l'histoire des personnages et la sienne, il maintenait pourtant cette opposition entre acadianité et universalité :

J'ai toujours écrit [...] Mon père aussi, était écrivain. Il a publié quatre ou cinq livres sur l'Histoire contemporaine de l'Acadie. Ma mère, elle, était musicienne et lisait beaucoup. " Mais cet article s'achève par les mots suivants: "Pour moi, c'est très important [...] de tenir un propos "universel" qui s'adresse à

23 Gérald Leblanc, op. cit., p. 93.

24 Jacques Savoie. Entre l'eau et la terre*, Publireportage, La presse, 12 novembre 1988, p. 8. 
76

l'humain en chaque lecteur, dans un monde où les moyens de communication ont fait disparaitre les distances. ${ }^{25}$

Son roman montre cependant que ce propos universel s'ancre dans une acadianité bien réelle. Mais il faut en même temps, semble-t-il que son expression ne donne pas prise à la folklorisation. D'où ces stratégies qui consistent à masquer au lecteur québécois les signes de l'acadianité et à dérouter le lecteur acadien de la leçon traditionnelle du récit acadien.

Je crois que Réginald Martel a implicitement compris cela quand il écrit: "Mais il y a plus et mieux. Jacques Savoie est allé chercher à New York, en passant par le Nouveau-Brunswick, un personnage qui donne au récit une singulière intensité ciramatique." ${ }^{26}$ Laisserait-il entendre que cette "singulière intensité dramatique", ce "plus et [ce] mieux" des Portes toumantes seraient dus à une certaine américanité acadienne? Ne touche-t-on pas ici à ce qui fait la différence entre le texte acadien et le texte québécois pour qui l'Amérique n'est jamais une donnée tout à fait naturelle mais souvent une difficulté de l'imaginaire et de ses représentations? Et c'est par là, cróyons-nous, que tout québécois qu'il soit, l'espace de Blaudelle est travaillé par le modèle acadien qui lui facilite l'ouverture de ses frontières. Sans être niée, l'acadianité n'est pas à l'avant-scène. On y entre et on en sort, comme à travers les Portes tournantes. Distanciée mais subtilement présente sous ses déguisements, elle a pour effet de faire de l'espace québecquois (symbole d'homogénéité par excellence) un lieu d'échange avec l'étranger, en y introduisant la capacité acadienne de concevoir l'enracinement comme une errance selon la formule d'Émile Ollivier ${ }^{27}$.

Quant à savoir quel pourrait être l'effet de l'interférence québécoise sur la lecture acadienne des Portes toumantes, il s'agit là d'une question qui, je crois, excède ma compétence de lecteur!

25 Jean Chapdelaine Gagnon, "L'écriture est une histoire de coeur pour Jacques Savoie $"$ Le devoir, $1^{\text {er }}$ octobre 1988, p. D-1.

26 Réginald Martel, op. cit.

27 Ghila Benesty-Sroka, Identités nationales. Interviews, Montréal, Éditions de la pleine lune, 1990, p. 131. 